\title{
Chapter 20 \\ Collaborative Innovation Between Shenzhen Municipal Government and Tsinghua University
}

\author{
Feiyu Kang
}

Global megatrends, such as the rise of frontier technologies, dramatic growth of information, sociopolitical landscape changes, and demographic trends, create a continuous need for transition. The increasing complexity of this phenomenon brings calls for ecosystem orchestration, which requires collaboration. The People's Republic of China (PRC), in particular, has been heavily investing in Artificial Intelligence (AI) and innovation to reduce gaps in basic research breakthroughs and high-end product development, and to further leverage its position as a manufacturing powerhouse. ${ }^{1}$

The PRC Government's commitment to support AI and Research and Development (R\&D) is articulated in its current five-year National Development Plan (2016-2020) as well in its National Medium- and Long-Term Plan for the Development of Science and Technology (2006-2020). ${ }^{2}$ The country has been pouring huge investments into $\mathrm{R} \& \mathrm{D}$ and is endeavoring to boost innovation.

\section{Issues and Challenges}

However, despite huge efforts and investments, the PRC faces challenges and roadblocks hindering its pursuit to become an innovation leader globally. It has faced deficits in terms of ensuring that its education system is prepared for an automationdriven economy, especially in fulfilling the rising need for skilled labor in smart manufacturing. ${ }^{3}$

\footnotetext{
${ }^{1}$ He, Y. 2017, June. How China is Preparing for an AI-powered Future, Wilson Briefs 2017. ${ }^{2}$ Ibid.

${ }^{3}$ Ibid.

F. Kang $(\bowtie)$

Department of Materials Science and Engineering, Graduate School at Shenzhen, Tsinghua University, Shenzhen, People's Republic of China

e-mail: fykang@tsinghua.edu.cn

B. Panth and R. Maclean (eds.), Anticipating and Preparing for Emerging Skills and Jobs, Education in the Asia-Pacific Region: Issues, Concerns and Prospects 55, https://doi.org/10.1007/978-981-15-7018-6_20
} 
While the PRC has one of the largest pools of scientific and technological talents globally, ${ }^{4}$ the country has very few leading AI scientists capable of producing worldleading research. Unlike more developed and innovative nations such as Germany, the PRC's training system is not yet well-aligned with the latest needs of markets and with technological trends. The PRC has also not yet successfully cultivated a dynamic culture of innovation and is experiencing challenges in attracting and maximizing its talents. Under its current system, scientific and technological engineers do not enjoy any real benefits from being innovative. ${ }^{5}$

The PRC also needs to catch up with more developed countries in some core technologies, as it remains dependent on other countries for key and innovative components of its manufacturing output (e.g., high-performance circuits and infrastructure software). ${ }^{6}$ Similarly, it has a limited number of truly innovative and globally competitive companies. Most Chinese Small and Medium-sized Enterprises (SMEs) have been focusing their innovation efforts on generic products at the bottom of the value chain.

\section{Proposed Solutions}

Nevertheless, the PRC's innovation ecosystem has grown and improved steadily in recent years as the country is tapping the potential of higher education and research institutes for cultivating innovation, as evidenced by increased spending on and government support for basic research and independent projects. ${ }^{7}$ The country is also rapidly developing hi-tech parks, and independent innovation demonstration zones are growing fast. Data from 2016 show that 130 such parks and zones, which are less than $1 \%$ of the PRC's territory, account for nearly $40 \%$ of R\&D investment by all the country's enterprises, as well as $32.8 \%$ of revenue from sales of new products. ${ }^{8}$

The Chinese innovation ecosystem is also geared toward market-oriented technological innovations and commercialization of technology. ${ }^{9}$ More forward-looking companies have established engineering centers. Technology innovation partnerships among business and universities in the PRC are also becoming more diverse and business-driven. As such, funding support for entrepreneurship and innovation has also been growing and diversifying. To manage the momentum, the PRC Government has also set up regulatory mechanisms to protect intellectual property rights.

\footnotetext{
${ }^{4}$ World Economic Forum. 2016. China's Innovation Ecosystem. Retrieved from http://www3.wef orum.org/docs/WEF_GAC_On_China_Innovation_WhitePaper_2016.pdf.

${ }^{5}$ Ibid.

${ }^{6}$ Ibid.

${ }^{7}$ Ibid.

${ }^{8}$ Ibid.

${ }^{9}$ Ibid.
} 
It is noteworthy that, traditionally, there has been a disconnect between academic and industrial research. To address this, Chinese universities are increasingly pursuing exciting joint projects with enterprises. Such projects and endeavors include setting up technology enterprises, technology parks, and incubator programs. These universities have also been engaging entrepreneurs and organizations with local resources. $^{10}$

\section{Good Practices}

\section{Shenzhen Innovation Ecosystem}

The PRC's AI innovation ecosystem, in particular, has been burgeoning as the nation's major technology hubs support researchers, entrepreneurs, and investors. In 2016, three cities dominated the AI sector: Beijing, home to 242 AI companies; Shanghai with 112 AI companies; and Shenzhen with 93 AI companies. ${ }^{11}$

Shenzhen in Guangdong Province, PRC, is a very vibrant and enabling environment for start-ups pursuing technological innovations. Located near Hong Kong, China, the city of Shenzhen has two of the top 50 global AI start-ups: iCarbonX, an AI-biotech start-up that uses algorithms to analyze genomic, physiological, and behavioral data to provide customized health advice; and UBTECH Robotics, the first company in the PRC to commercialize humanoid robots that can interact with human tools and environments. Shenzhen is also a major manufacturing region for industrial robots, producing more than $\$ 11.4$ billion worth of robots in $2016 .{ }^{12}$

As the first National Innovation City and the first Special Economic Zone (SEZ) established in the country, Shenzhen has benefited from government policies supporting market openness, entrepreneurship, and R\&D collaboration between universities and firms. Initially a fishing village, Shenzhen had no academic institutions. The PRC Government anticipated that the lack of higher education and research institutions would hinder the industrial development of Shenzhen, so it invited leading universities to set up campuses and/or research bases in the city. ${ }^{13}$

The Shenzhen municipal government also notably established a University Virtual Campus (UVC) concept in 2000 to increase cooperation with existing university partners and encourage more higher education and research institutions to set up in Shenzhen. They provided incentives (e.g., free office space and infrastructure for

\footnotetext{
${ }^{10}$ Ibid.

${ }^{11}$ He, Y. 2017, June. How China is Preparing for an AI-powered Future, Wilson Briefs 2017. Retrieved from https://www.wilsoncenter.org/sites/default/files/how_china_is_preparing_for_ai_ powered_future.pdf.

${ }^{12}$ Ibid.

${ }^{13}$ Chen, K. and Kenney, M. 2007. Universities/Research Institutes and Regional Innovation Systems: The Cases of Beijing and Shenzhen. World Development, 35(6), pp. 1056-1074. Retrieved from https://pdfs.semanticscholar.org/15e0/43a1635dd0a85dac6df73590f8af708385b3.pdf.
} 
2 years, subsidized amenities) to universities and research institutions that would establish branches at the UVC. ${ }^{14}$ The incentive mechanism was so attractive that within 5 years, 43 universities and research institutions, including five universities in Hong Kong, China, and one university in France (Centrale Lyonais), put up branches at Shenzhen's UVC. ${ }^{15}$ Aside from being a successful leading education hub, Shenzhen has also become successful in producing research and enabling academeindustry linkages. In just 4 years, more than 120 high-tech enterprises were established by the universities and more than 100 research projects from the universities were transferred to industry. ${ }^{16}$

\section{Tsinghua-UC Berkeley Shenzhen Institute}

The Graduate School at Shenzhen, Tsinghua University (GSSTU) was jointly founded by Tsinghua University and the Shenzhen municipal government in 2001 to cultivate top-level professionals and to carry out scientific and technological innovations. Directly affiliated with Tsinghua University in Beijing, GSSTU plays an important role in Tsinghua's commitment to achieve international prominence.

In 2014, Tsinghua University signed a memorandum of understanding with the University of California, Berkeley for the establishment of the Berkeley-Tsinghua Alliance for Transformational Technology Research and Education in Shenzhen. The Tsinghua-UC Berkeley Shenzhen Institute (TBSI) is a joint research and educational collaboration among UC Berkeley, Tsinghua University, and the Shenzhen municipal government to promote research collaboration and graduate student education. TBSI provides a unique global platform for transformational, translational, and transdisciplinary research and education for future world leaders in science and technology. The program's objective is to prepare global leaders to address societal challenges through immersion programs in the PRC and the United States. ${ }^{17}$

TBSI's research platforms are organized into three transdisciplinary centers to foster critical fields of research that address societal needs and global challenges: (i) Environmental Science and New Energy Technology, (ii) Information Technology and Data Science, and (iii) Precision Medicine and Healthcare. TBSI also offers a 2.5-year dual-degree Master of Engineering program, which is cotaught and takes place in both UC Berkeley and Tsinghua University (Phase 1 starts at Shenzhen, Phase 2 at UC Berkeley, and Phase 3 at Tsinghua University). ${ }^{18}$

\footnotetext{
${ }^{14}$ Ibid.

${ }^{15}$ Ibid.

${ }^{16}$ Ibid.

${ }^{17}$ UC Berkeley. (n.d.). Tsinghua-Berkeley Shenzhen Institute. Retrieved from https://tbsi.berkeley. edu/.

${ }^{18}$ Ibid.
} 
Tsinghua University aims to use the positive experiences from TBSI to establish a Tsinghua Global Campus at Shenzhen, with the goal of tripling the size of Tsinghua Shenzhen Campus by 2025. The Global Campus is envisioned to build world-class transformative research, education, and innovation partnerships to nurture transformational technology and social science research that addresses global challenges, builds new capabilities, and co-creates markets and ecosystems.

\section{Application of These Good Practices}

The success of the Shenzhen Innovation Ecosystem and TBSI highlights the importance of taking advantage of favorable market conditions by timely aligning government policies and tapping strategic partnerships toward achieving development goals. The PRC Government's incentives and enabling innovation ecosystem were effective in attracting foreign talents and convincing renowned institutions to partner with its universities. International collaborations such as TBSI enhance the PRC innovation system, as they capacitate local talents and advance research through knowledge transfer/exchange and funding opportunities. These partnerships, through dual-exchange programs and transnational education, are also notably successful in terms of enabling local universities to upgrade their programs' curricula and cultivate advanced and higher level skills among their students.

University-research-industry linkages have also generally proven to be successful in achieving innovation-driven endeavors in the PRC. This is evidenced by the large number of firms and start-ups from such linkages that have grown into large businesses. Key PRC technology firms such as Lenovo, Tongfang, and Founder started as university-research-industry projects. ${ }^{19}$ However, it is important to note that their successes are due to close relationships between universities and industries. Such partnerships also strategically embark on business-driven technological innovations and commercialization of technology, unlike conventional collaborations that involve mostly knowledge exchange and funding.

As the PRC's policies and innovation ecosystem have been widely successful in tapping the capabilities of its researchers, universities, and industries, developing countries may find the university-industry-linkage model useful. While most countries consider some separation between universities as healthy, the PRC model holds good potential in the context of gearing economies to be more knowledge-based and innovation-driven. This approach, however, would entail proactive and enabling government policies as well as strong willingness from universities and businesses to engage in joint ventures.

\footnotetext{
${ }^{19}$ Chen, K. and Kenney, M. 2007. op. cit.
} 


\section{Implication for the Future}

The pace of technological advancements is anticipated to rapidly change the needs of the labor market and education systems globally. Many countries, especially the PRC, have been preparing for such developments by heavily investing in endeavors that will enable them to become leaders in science, technology, and innovation. Universities, in particular, have been tapped as critical players in this race.

To keep up with dynamic changes in research and curricula, education systems need to ensure that teachers are well equipped to cope. Learning methodologies are expected to adapt quickly to rapid technological progress and ensure responsiveness to the needs of the labor market, and educators need to have adequate competencies to teach these. Emerging fields and disciplines brought by technological advancements will also require higher level skills and create new demand for curricular offerings/programs. This will also require more efforts in adult education, retooling, and/or upskilling, given the expected effects of disruptive technologies on jobs (i.e., automation, demand for new jobs). For countries to fully benefit from internationalization and university-industry endeavors, their education systems also need to be able to timely address these needs.

\section{Conclusion}

With the onset of the Fourth Industrial Revolution, universities have a critical role in the creation of knowledge-based and innovation-driven economies. As such, innovative and collaborative practices that sustain the excellence and relevance of universities will be important to compete globally. Furthermore, education systems need to prepare both teachers and learners and ensure that they have the right competencies to thrive in a very dynamic labor and skills landscape. To achieve these, governments need to be proactive in creating an ecosystem that will encourage industry and academe to embark on breakthrough education and R\&D ventures.

Link to presentation material: https://events.development.asia/materials/201 71212/collaborative-innovation-between-shenzhen-city-government-and-tsinghuauniversity.

\section{References}

Chen, K., \& Kenney, M. (2007). Universities/research institutes and regional innovation systems: The cases of Beijing and Shenzhen. World Development, 35(6), 1056-1074. Retrieved from https://pdfs.semanticscholar.org/15e0/43a1635dd0a85dac6df73590f8af708385b3.pdf. 
He, Y. (2017, June). How China is preparing for an AI-powered future. Wilson Briefs 2017. Retrieved from https://www.wilsoncenter.org/sites/default/files/how_china_is_preparing for_ai_powered_future.pdf.

University of California Berkeley. (n.d.). Tsinghua-Berkeley Shenzhen Institute. Retrieved from https://tbsi.berkeley.edu/.

World Economic Forum. (2016). China's innovation ecosystem. Retrieved from http://www3.wef orum.org/docs/WEF_GAC_On_China_Innovation_WhitePaper_2016.pdf.

The views expressed in this Chapter are those of the authors and do not necessarily reflect the views and policies of the Asian Development Bank (ADB) or its Board of Governors or the governments they represent.

ADB does not guarantee the accuracy of the data included in this Chapter and accepts no responsibility for any consequence of their use. The mention of specific companies or products of manufacturers does not imply that they are endorsed or recommended by ADB in preference to others of a similar nature that are not mentioned.

By making any designation of or reference to a particular territory or geographic area, or by using the term "country" in this Chapter, ADB does not intend to make any judgments as to the legal or other status of any territory or area.

This work is available under the Creative Commons Attribution 3.0 IGO license (CC BY 3.0 IGO) https://creativecommons.org/licenses/by/3.0/igo/. By using the content of this Chapter, you agree to be bound by the terms of this license. For attribution, translations, adaptations, and permissions, please read the provisions and terms of use at https://www.adb.org/terms-use\#openac cess.

This CC license does not apply to non-ADB copyright materials in this Chapter. If the material is attributed to another source, please contact the copyright owner or publisher of that source for permission to reproduce it. ADB cannot be held liable for any claims that arise as a result of your use of the material.

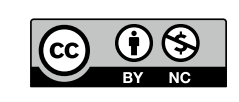

